\title{
Switching from molecular to bulklike dynamics in electronic relaxation of a small gold cluster
}

\author{
Jörg Stanzel,,${ }^{1,2}$ Matthias Neeb, ${ }^{1, *}$ Wolfgang Eberhardt, ${ }^{3}$ Polina G. Lisinetskaya, ${ }^{4}$ Jens Petersen,${ }^{4}$ and Roland Mitrićc ${ }^{4, \dagger}$ \\ ${ }^{1}$ Helmholtz-Zentrum Berlin, Wilhelm-Conrad-Röntgen Campus Adlershof, Elektronenspeicherring BESSY II, \\ Albert-Einstein-Strasse 15, D-12489 Berlin, Germany \\ ${ }^{2}$ Inventux Technologies AG, Wolfener Strasse 23, D-12681 Berlin, Germany \\ ${ }^{3}$ Technische Universität Berlin, IOAP, Strasse des 17, Juni 135, 10623 Berlin, Germany \\ ${ }^{4}$ Freie Universität Berlin, Fachbereich Physik, Arnimallee 14, D-14195 Berlin, Germany
}

(Received 30 June 2011; published 10 January 2012)

\begin{abstract}
We have investigated the ultrafast electronic relaxation of $\mathrm{Au}_{7}^{-}$using time-resolved photoelectron spectroscopy combined with first-principles simulations of the excited-state dynamics. Unlike previous findings, which have demonstrated molecularlike excited-state relaxation in $\mathrm{Au}_{7}^{-}$at low excitation energy $(1.56 \mathrm{eV})$, we show here that excitation with $3.12 \mathrm{eV}$ leads to bulklike electronic relaxation without a considerable change of geometry. The experimental findings are fully supported by theoretical simulations, which reveal a bulklike electron-hole relaxation mechanism in a far band-gap cluster. Our findings demonstrate that small gold clusters in the sub-nm size range can exhibit either molecularlike or bulklike properties, depending on the excitation energy.
\end{abstract}

DOI: 10.1103/PhysRevA.85.013201

PACS number(s): 36.40.-c, 31.15.A-, 78.47.jd, 87.15.ht

\section{INTRODUCTION}

The size reduction of materials leading to small clusters in the nonscalable size regime $(<2 \mathrm{~nm})$ drastically changes the electronic, structural, and chemical properties. This makes clusters promising building blocks for new optical and catalytic materials. From the fundamental point of view, the reduction of size leads to a transition from the bulk band electronic structure to discrete molecularlike energy levels. Therefore, the question can be raised as to whether, in a cluster of a given size, the effects arising from both the molecularlike as well as from the bandlike electronic structure can be observed. Due to the fact that in small gold clusters, as a consequence of relativistic effects, the energy gap between the $5 d$ and $6 s$ atomic states is particularly low [1], they exhibit in the low-energy regime both discrete energy levels derived from the $6 s$ atomic states as well as bandlike levels derived from the $5 d$ atomic states. Therefore, it is expected that both molecularlike as well as bulklike dynamical behavior and electronic relaxation can be observed in gold clusters under properly chosen conditions. This opens perspectives to tune the optical response of cluster-based plasmonic and photonic materials by exploiting the interplay between the ultrafast electronic relaxation processes and the photoinduced nuclear dynamics.

Time-resolved photoelectron spectroscopy (TRPES) on mass-selected clusters offers the unique opportunity to study ultrafast relaxation [2-5], since it is sensitive both to nuclear motion as well as to changes in the electronic structure. In recent years, TRPES has been employed to investigate excited-state dynamics in a variety of systems, ranging from organic molecules and biochromophores [6,7] to metallic and molecular clusters $[4,8,9]$. For open $d$-shell transitionmetal clusters with a large excited-state density, it has been shown that an ultrafast electronic relaxation takes place $[2,10]$

\footnotetext{
*neeb@bessy.de

†mitric@zedat.fu-berlin.de
}

involving a rapid change of the electronic configuration via nonadiabatic coupling [11]. A completely different scenario is observed if electronic relaxation is suppressed, which occurs when the populated excited state is energetically well separated from other states, as in the case for clusters of metals with a closed $d$ shell [12-14]. In particular, the electronic structure of small gold clusters is characterized by isolated low-lying electronic states, which arise from the combination of $6 s$ atomic levels, followed by a much denser manifold of $5 d$-derived states $[15,16]$. As we have previously observed, the excitation of low-lying states in $\mathrm{Au}_{n}^{-}(n=5-8)$ clusters leads to a molecularlike isomerization involving only a single excited electronic state [9].

In this paper, we present a joint experimental and theoretical study of the electronic relaxation in the small anionic gold cluster $\mathrm{Au}_{7}^{-}$. The chosen excitation energy of $3.12 \mathrm{eV}$ is resonant with a dense manifold of $5 d$-derived electronic states. Excitation of these states leads to ultrafast electronic relaxation without a significant change of the nuclear geometry. We show by experimental TRPES, as well as by simulations of laserinduced excited state dynamics, that the relaxation is driven by strong nonadiabatic coupling between many close-lying excited states. In terms of a single-particle picture, this corresponds to electron-hole relaxation caused by electron-phonon coupling. This behavior is similar to the electronic relaxation observed in bulk metals, but takes place on a significantly longer time scale of several hundreds of femtoseconds. In this sense, we demonstrate that in addition to the molecularlike dynamics observed previously [9], small gold cluster anions can also exhibit bulklike relaxation depending on the used excitation energy. Due to the far band gap of the investigated $\mathrm{Au}$ cluster, no secondary electrons are generated during electron-hole relaxation, in contrast to hot-electron relaxation in narrow band gap clusters such as $\mathrm{Pt}_{n}^{-}$or $\mathrm{Pd}_{n}^{-}[2,10]$.

The paper is structured as follows. In Sec. II, the experimental setup is briefly presented, and the theoretical approach used for the computation is outlined. In Sec. III, the experimental and theoretical results on the TRPES of $\mathrm{Au}_{7}^{-}$are discussed. Finally, conclusions and outlook are given in Sec. IV. 


\section{EXPERIMENTAL SETUP AND COMPUTATIONAL DETAILS}

The experimental setup is described in detail in Refs. [8,9]. Briefly, cold gold cluster anions $(T \sim 250 \mathrm{~K})$ are produced in a pulsed laser vaporization source by aggregation during supersonic expansion within a $\mathrm{He}$ carrier gas. The cluster anions are mass separated by a time-of-flight mass spectrometer and decelerated before irradiation with two time-delayed fs-laser pulses. In a two-color pump-probe experiment, the dynamics of the photoexcited cluster anions is probed by recording a series of photoelectron spectra with varied time delay between pump and probe pulse. The kinetic energy of the detached electrons is analyzed by a magnetic bottle time-of-flight photoelectron spectrometer. We employed a mode-locked Ti:sapphire laser consisting of an oscillator, a multipass amplifier, and a prism compressor (pump pulse duration of $40 \mathrm{fs}$ FWHM, energy per pulse of $<1 \mathrm{~mJ}$, and spectral bandwidth of $40 \mathrm{meV}$ FWHM). The second harmonic of the Ti:sapphire laser served as a pump $(3.12 \mathrm{eV})$ and the fundamental served as a probe pulse $(1.56 \mathrm{eV})$. A cross-correlation pulse duration of $60 \mathrm{fs}$ has been measured close to the interaction zone inside the vacuum.

Theoretically, the laser-driven dynamics and TRPES have been simulated using our field-induced surface-hopping method (FISH), which has been described in detail previously [17-19]. Briefly, it is based on the propagation of classical molecular dynamics trajectories "on the fly" in a manifold of excited electronic states, which are here described using the time-dependent density functional theory (TDDFT) with the gradient-corrected Perdew-Burke-Ernzerhof (PBE) exchangecorrelation functional [20]. For gold, we use the triple- $\zeta$ valence plus polarization atomic Gaussian basis set (TZVP) [21], and the relativistic 19 electron effective core potential [22]. In order to simulate both bound-state dynamics and the photoionization, the manifold of 50 bound electronic states in $\mathrm{Au}_{7}^{-}$has been augmented by a discretized continuum of 120 states according to the procedure described in Ref. [18]. Along each trajectory, the time-dependent Schrödinger equations for all bound and continuum states,

$$
\begin{gathered}
i \hbar \dot{c}_{I}^{(N)}(t)=E_{I}(\mathbf{R}(t)) c_{I}^{(N)}(t)-\sum_{K}^{\text {bound }}\left[\boldsymbol{\varepsilon}(t) \cdot \boldsymbol{\mu}_{I K}(\mathbf{R}(t))+i \hbar D_{I K}(\mathbf{R}(t))\right] c_{K}^{(N)}(t), \\
i \hbar \dot{c}_{J}^{(N-1)}(E, t)=\left[E_{J}^{(N-1)}(\mathbf{R}(t))+E\right] c_{J}^{(N-1)}(E, t)-\sum_{K}^{\text {bound }} \boldsymbol{\varepsilon}(t) \cdot \boldsymbol{\mu}_{J K}(\mathbf{R}(t), E) c_{K}^{(N)}(t),
\end{gathered}
$$

are solved simultaneously. In these equations, $c_{I}^{(N)}$ represents the amplitude of the $I$ th bound electronic state in the total electronic wave function,

$$
|\Psi(\mathbf{R}(t))\rangle=\sum_{I} c_{I}^{(N)}(t)\left|\Psi_{I}(\mathbf{R}(t))\right\rangle
$$

while $c_{J}^{(N-1)}$ is the coefficient for the $J$ th discretized continuum state with the energy of the ionized species $E_{J}^{(N-1)}$ and the photoelectron kinetic energy $E$. The individual trajectories are allowed to switch between the states according to transition probabilities, which are calculated fully quantum mechanically based on the $c_{I}^{(N)}(t)[17,19]$. The nonadiabatic couplings $D_{I K}$ as well as the transition dipole moments $\boldsymbol{\mu}_{I K}$ are obtained from the TDDFT method by defining an auxilliary wave function for each excited electronic state, consisting of all single particle-hole excitations in the manifold of the Kohn-Sham (KS) orbitals $[23,24]$. It has the form

$$
\left|\Psi_{I}\right\rangle=\sum_{p h} c_{p h}^{I}(t) a_{p}^{\dagger} a_{h}\left|\Phi_{K S}\right\rangle
$$

where $a_{p(h)}^{\dagger}$ and $a_{p(h)}$ represent fermionic creation and annihilation operators for particles and holes, respectively, $\left|\Phi_{K S}\right\rangle$ is the ground-state KS determinant, and the coefficients $c_{p h}^{I}$ are determined from the TDDFT eigenvectors [23]. The solution of the coupled sets of Eqs. (1) using the experimental pump and probe pulses yields the population of the ionized states, which is employed to calculate the TRPES signal

$$
S\left(t_{d}, E\right)=\frac{1}{N_{\text {traj }}} \sum_{\text {traj }} \sum_{I}\left|c_{I}^{(N-1)}(E, t \rightarrow \infty)\right|^{2}
$$

by averaging over the ensemble of $N_{\text {traj }}$ trajectories.

\section{RESULTS AND DISCUSSION}

The theoretical absorption spectra for the two lowest isomers (isomers 1 and 2 in the following) of $\mathrm{Au}_{7}^{-}$are presented in Figs. 1(a) and 1(b). Above $2 \mathrm{eV}$, the dense manifold of electronic states can be divided into three energetic regions (I, II, and III in Fig. 1). The character of dominant excitations in the representative states of these three manifolds is illustrated in Figs. 1(c)-1(e). The electronic relaxation of $\mathrm{Au}_{7}^{-}$has been investigated by exciting the cluster mainly into the energy region III (cf. Fig. 1) using a pump energy of $3.12 \mathrm{eV}$ and measuring TRPES. Experimental and theoretical TRPE spectra are presented in Fig. 2. At $t=0.1 \mathrm{ps,} \mathrm{two}$ photodetachment peaks $\left(X\right.$ and $\left.X^{\prime}\right)$ exhibiting different timedependent behavior appear in the experimental spectrum at a kinetic energy of 1.3 and $0.8 \mathrm{eV}$, respectively [cf. Fig. 2(a)]. The intensity of the peak $X$ decays almost exponentially with a time constant $\tau_{X}=0.6 \pm 0.1 \mathrm{ps}$, whereas the intensity of peak $X^{\prime}$ decays with a time constant $\tau_{X^{\prime}}=1.6 \pm 0.2 \mathrm{ps}$ [cf. Fig. 2(a)]. The time evolution of the integrated intensities 


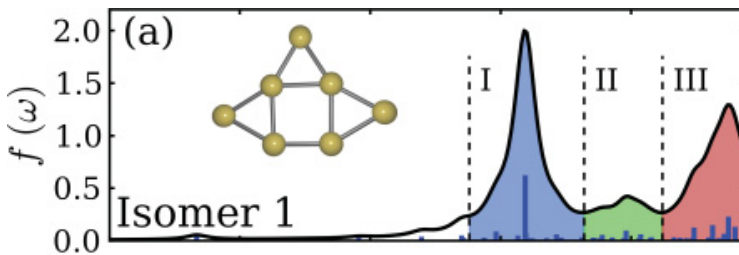

(c)
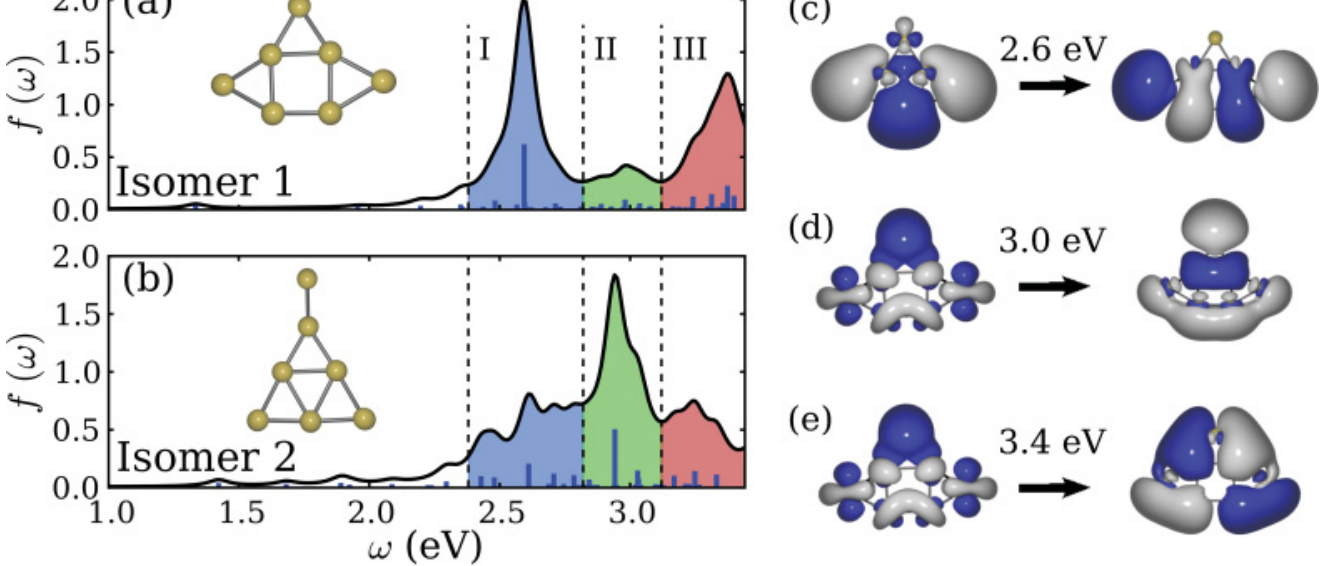

FIG. 1. (Color online) (a), (b) Calculated absorption spectra for the two lowest energy isomers of $\mathrm{Au}_{7}^{-}$. (c)-(e) Kohn-Sham orbitals corresponding to the dominant excitations for characteristic transitions of the isomer 1 in the energy regions (c) I, (d) II and (e) III.

of the three energy regions present in the TRPE spectrum is provided in Fig. 3.

The simulated TRPES shown in Fig. 2(b) has been obtained by superposing the spectra of isomers 1 and 2 with equal weights since both isomers are energetically degenerate [25]. The overall agreement between theoretical and experimental TRPES is very good. Both peaks $X$ and $X^{\prime}$ are situated at the same energy as in the experiment, which gives evidence for electron relaxation without energy transfer to the nuclear system. The slow intensity decrease indicates to a strong coupling of various excited states. The mean energy of the photoemission peaks only slightly changes with time. The overall loss of photoelectron intensity after $1 \mathrm{ps}$ time is due to the fact that $X$ and $X^{\prime}$ disappear from the Franck-Condon region of the relaxed anionic states.

In order to address the mechanism of the electronic relaxation and to calculate the lifetime of the photoexcited state, we show in Fig. 4(a) the simulated electronic-state population dynamics of isomer 1 of $\mathrm{Au}_{7}^{-}$induced by the experimental pump pulse, which leads to the population of electronic states from region III by $\sim 50 \%$ (cf. Fig. 1). Ionization of these states to the ground state of the neutral species produces photoelectrons of $\sim 1.3 \mathrm{eV}$ kinetic energy, giving rise to the peak $X$ in the TRPES (cf. Fig. 2). Furthermore, the first excited neutral state $(\sim 0.8 \mathrm{eV}$ above the ground state) also can be reached by the probe pulse at the initial stage of the dynamics, leading to photoelectrons with low kinetic energies of $\sim 0.3 \mathrm{eV}$. After excitation by the pump pulse, the population from region III decays into lower-lying states (region II) with a time constant of $550 \mathrm{fs}$, accompanied by the broadening of peak $X$ and the decay of the maximum intensity. The theoretical time constant agrees well with the experimental one of $\tau_{X}=$ $600 \pm 100$ fs. Notice that simulated TRPES decays slower than the experimental one, which is attributed to difficulties in the calculation of the correct photoionization cross sections. After $400 \mathrm{fs}$, low-lying states from region I also start to be populated, but essentially no population is transferred to the ground state within 1000 fs. Similarly, excitation of the isomer 2 also populates states at about $3 \mathrm{eV}$ excitation energy, followed by decay to lower-lying excited states, as shown in Fig. 5(a). However, since the electron detachment energy of this isomer is higher than that of the first one, the released photoelectrons exhibit lower kinetic energies of around $0.8 \mathrm{eV}$, thus giving rise to the TRPES peak $X^{\prime}$.

Additional insight into the relaxation process can be gained from the ensemble-averaged time-dependent electronic-state

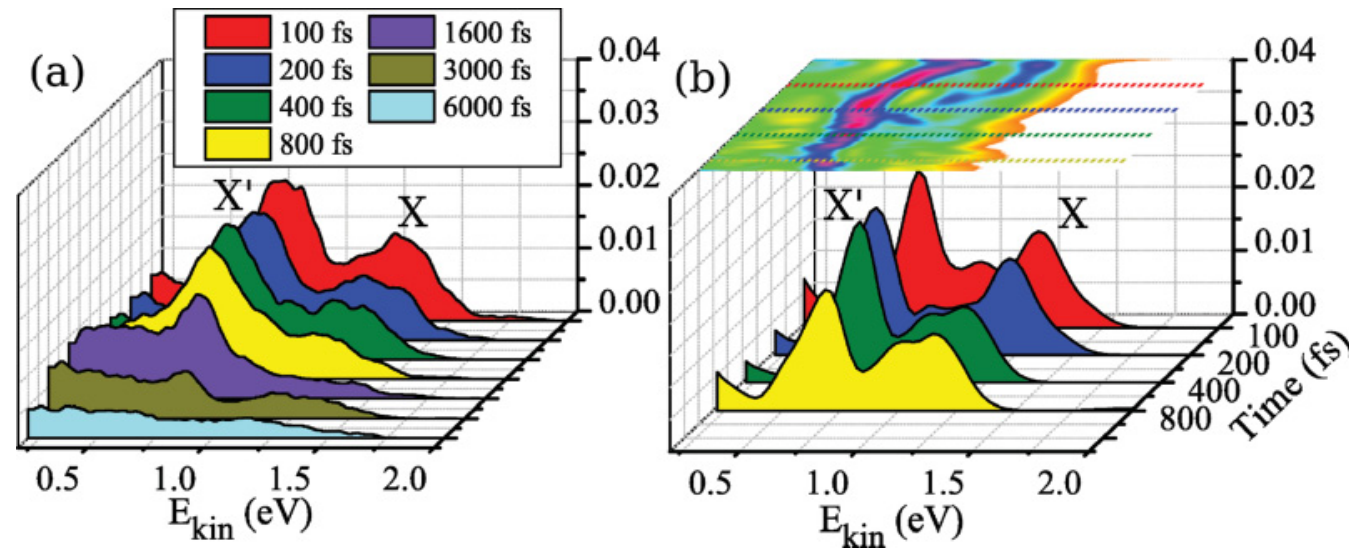

FIG. 2. (Color online) (a) Experimental and (b) simulated TRPES of $\mathrm{Au}_{7}^{-}$with $h v_{\text {pump }}=3.12 \mathrm{eV}$ and $h v_{\text {probe }}=1.56 \mathrm{eV}$. The full simulated TRPES with a time resolution of $20 \mathrm{fs}$ is shown in (b) as a two-dimensional plot on the top. 


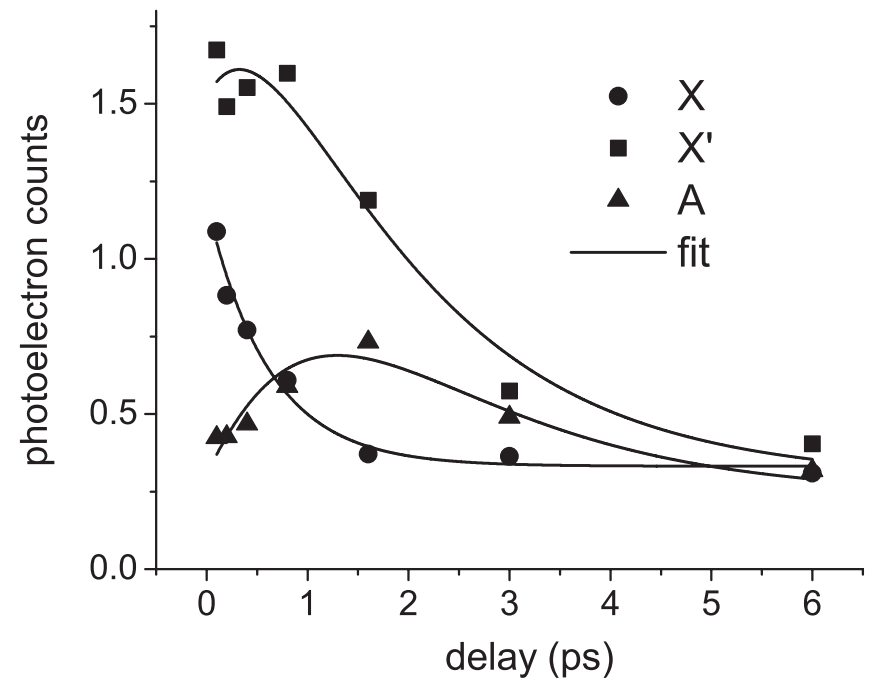

FIG. 3. Integrated experimental photoelectron yield of the three energy regions $X, X^{\prime}$, and $A$ (energy region $<0.5 \mathrm{eV}$ ) as a function of time. The photoelectron transients have been fitted by a model consisting of three coupled rate equations [26] numerically solved by the Runge-Kutta method (solid lines). This model provides exponential decay time constants for the intensity of $X, \tau_{X}=0.6 \pm 0.1 \mathrm{ps}$, and of $X^{\prime}, \tau_{X^{\prime}}=1.6 \pm 0.2 \mathrm{ps}$. The intensity of region $A$ increases up to 1.5 ps before decaying with $\tau_{A}=0.7 \pm 0.2$ ps. After $6 \mathrm{ps,} \mathrm{no}$ significant photoelectron intensity is visible in any of the three energy regions.

energies, which are presented for the isomer 1 in Fig. 4(b). The electronic states populated by the laser excitation lie in a bandlike manifold between 2.3 and $3.3 \mathrm{eV}$, with a mean energy separation between individual states of only $\sim 20$ $\mathrm{meV}$. Therefore, a strong coupling between these close-lying states occurs, leading to an ultrafast electronic-state population decay. As can be seen from Fig. 4(b), the average electronic energy decreases from $\sim 3.2$ to $2.9 \mathrm{eV}$ within $1 \mathrm{ps}$. This is
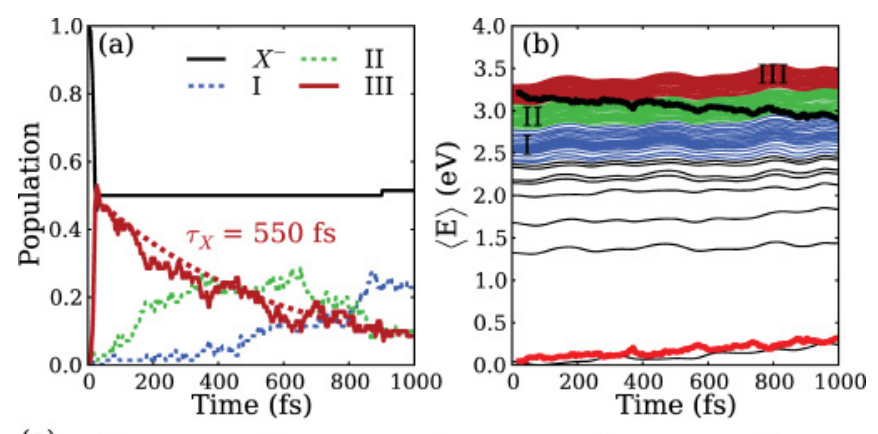

(c)

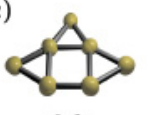

$0 \mathrm{fs}$

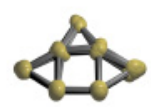

$250 \mathrm{fs}$

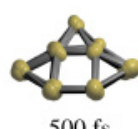

$500 \mathrm{fs}$

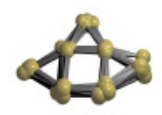

$750 \mathrm{fs}$

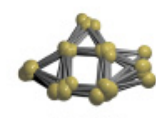

$1000 \mathrm{fs}$
FIG. 4. (Color online) (a) Excited state population dynamics of the isomer 1 of $\mathrm{Au}_{7}^{-}$for the ground state $X^{-}$and the states in excitation energy regions I, II, and III simulated using the FISH method with $h v_{\text {pump }}=3.12 \mathrm{eV}$. (b) Temporal evolution of the excited and ground electronic state energies averaged over the ensemble of trajectories. The energy regions I, II, and III are indicated in the graph. The averaged nuclear kinetic energies and electronic energies are also shown as thick red (light gray) and black lines.
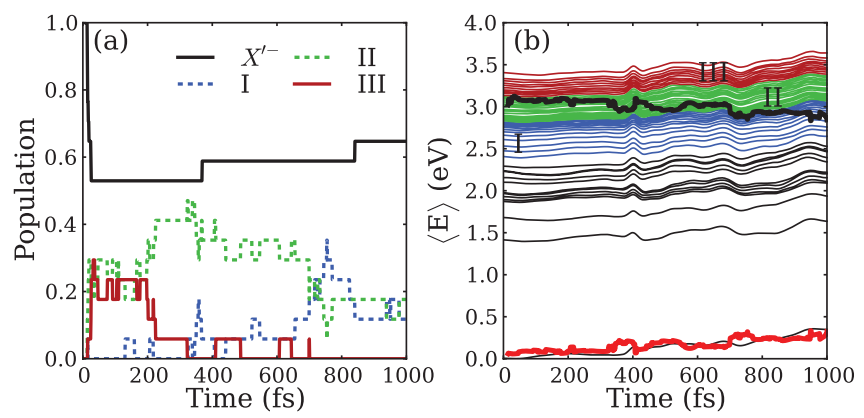

FIG. 5. (Color online) (a) Excited state population dynamics of the isomer 2 of $\mathrm{Au}_{7}^{-}$for the ground state $X^{\prime-}$ and the states in excitation energy regions I, II, and III simulated using the FISH method with $h v_{\text {pump }}=3.12 \mathrm{eV}$. (b) Temporal evolution of the excited and ground electronic state energies averaged over the ensemble of trajectories. The energy regions I, II, and III are indicated in the graph. The averaged nuclear kinetic energies and electronic energies are also shown as thick red (light gray) and black lines.

accompanied by energy transfer to the nuclear vibrational modes, which arises as a consequence of a large nonadiabatic coupling (electron-phonon coupling). The total nuclear kinetic energy increases correspondingly from almost zero to about $0.3 \mathrm{eV}$ [cf. Fig. 4(b)]. A very similar behavior is observed for the isomer 2 [cf. Fig. 5(b)]. The nuclear configuration, however, remains essentially the same and no large amplitude motion occurs (cf. snapshots in Fig. 4). Therefore, the excitedstate dynamics after $3.12 \mathrm{eV}$ excitation is mainly governed by changes in the electronic configuration rather than by nuclear motion. This behavior is very different to that observed after $1.56 \mathrm{eV}$ excitation, which leads to isomerization and photoinduced melting of $\mathrm{Au}_{7}^{-}$, as recognized by a strong time-dependent energy shift of the photodetachment peak in the TRPES [9].

Interestingly, as the electron and hole get closer to the highest occupied and lowest unoccupied molecular-orbital (HOMO and LUMO) levels, the electronic relaxation becomes slower, and the discrete nature of the electronic states begins to play a dominant role. Eventually, this leads to exceptionally long electronic lifetimes in small noble metal clusters, which are responsible, e.g., for their fluorescence. In contrast, when using a lower excitation energy which just bridges the HOMOLUMO energy gap, electron-hole relaxation cannot occur and the dynamics is dominated by nuclear motion, as observed for $\mathrm{Au}_{7}^{-}$and $\mathrm{Hg}_{n}^{-}$clusters at $1.5 \mathrm{eV}$ excitation energy $[9,12,13]$.

A complementary perspective on the relaxation mechanism can be obtained in terms of the electron-hole picture. Since in our simulations at each time step the full many-electron wave function consisting of numerous individual electron-hole excitations is available, it is possible to calculate the average electron and hole energies as a function of time. This has been performed by defining an effective electron-hole Hamiltonian based on the KS orbitals as

$$
\hat{H}_{\mathrm{eff}}=\epsilon_{F}-\sum_{h} \epsilon_{h} a_{h}^{\dagger} a_{h}+\sum_{p} \epsilon_{p} a_{p}^{\dagger} a_{p},
$$

where $\epsilon_{F}, \epsilon_{p}$, and $\epsilon_{h}$ are the Fermi energy, and the electron and hole energies, respectively. The average electron and hole 

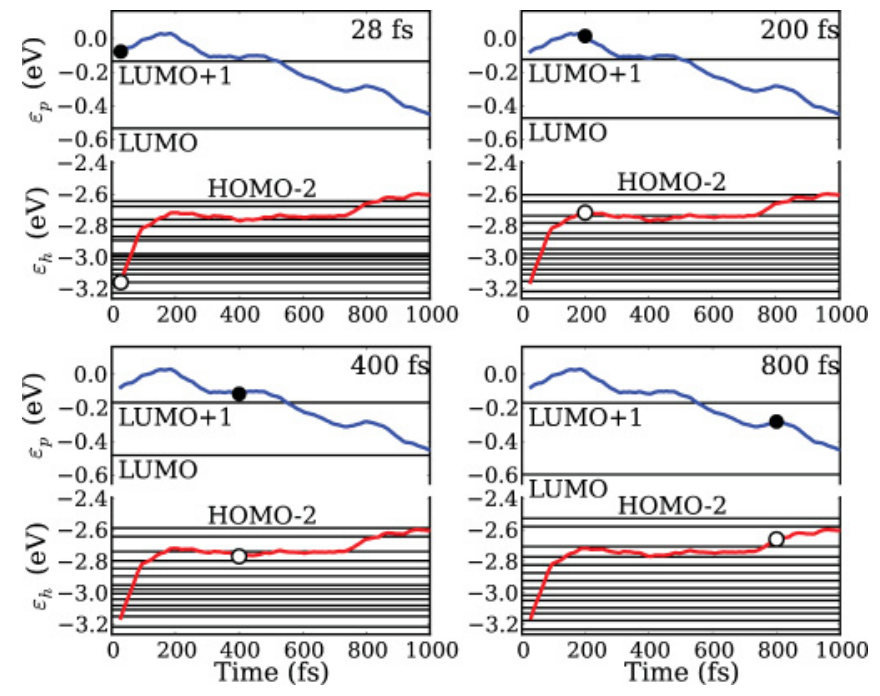

FIG. 6. (Color online) Time evolution of the hole (red, lower line) and electron (blue, upper line) energies. The instantaneous energies of the electron (filled circle) and hole (empty circle) for selected times are shown together with the energies of the Kohn-Sham orbitals (horizontal black lines) at the stated times.

energies can be calculated as expectation values of the effective Hamiltonian according to

$$
\begin{aligned}
\left\langle\epsilon_{p(h)}\right\rangle & =\left\langle\Psi(\mathbf{R}(t))\left|\sum_{p(h)} \epsilon_{p(h)} a_{p(h)}^{\dagger} a_{p(h)}\right| \Psi(\mathbf{R}(t))\right\rangle \\
& =\sum_{p(h)} \epsilon_{p(h)} \sum_{I}\left|c_{I}(t)\right|^{2}\left|c_{p h}^{I}(t)\right|^{2} .
\end{aligned}
$$

Their time evolution along the trajectories is presented in Fig. 6. The electron energy exhibits an overall drop of $\sim 0.4 \mathrm{eV}$ within 1 ps, modulated by low-frequency oscillations of the nuclear framework. This corresponds to relaxation of the electron from the LUMO+1 to the LUMO level of the system.
Complementary to that, the energy of the hole increases by about $0.5 \mathrm{eV}$. Due to the much higher density of states below the Fermi level, the relaxation of the hole is initially faster than that of the electron, but gets slower after $\sim 200 \mathrm{fs}$ when the hole reaches the region with lower density of states. Both the electron and the hole relax gradually towards the Fermi level (HOMO). This mechanism resembles in many respects the electron-phonon relaxation in bulk metals, but takes place on much longer time scale due to the finiteness of the system.

\section{CONCLUSION}

We have studied the relaxation dynamics of optically excited electronic states in $\mathrm{Au}_{7}^{-}$by experimental TRPES and FISH theoretical simulations. Our findings provide a detailed molecular mechanism of the electronic relaxation in $\mathrm{Au}_{7}^{-}$ and show that the dynamics of $d$-derived bandlike states is governed by nonadiabatic electronic relaxation, in contrast to $s$-derived molecularlike states, which exhibit isomerization and ultrafast decay to the ground state. Notice that no secondary electrons are generated in the proven single-electron single-hole relaxation pathway, which is in strong contrast to relaxation in bulk metals and narrow-band-gap clusters. Thus, small gold clusters with a size less than $\sim 2 \mathrm{~nm}$ can be controlled to exhibit either molecularlike or bulklike behavior, depending on the regime of electronic excitation. These properties are unique for systems in this size range and could be exploited for the development of cluster-based materials with a tailored optical response.

\section{ACKNOWLEDGMENTS}

We are grateful to the Deutsche Forschungsgemeinschaft (DFG) for financial support via the Emmy-NoetherProgramme (Project No. MI-1236) (P.G.L. and R.M.) as well as the DFG Projects No. FOR 1282 (J.P., R.M., M.N., and W.E.) and No. SPP 1391 (R.M.).
[1] P. Pyykkö, Angew. Chem. Int. Ed. 43, 4412 (2004).

[2] N. Pontius, P. S. Bechthold, M. Neeb, and W. Eberhardt, Phys. Rev. Lett. 84, 1132 (2000).

[3] A. Stolow, Annu. Rev. Phys. Chem. 54, 89 (2003).

[4] A. Stolow, A. E. Bragg, and D. M. Neumark, Chem. Rev. 104, 1719 (2004).

[5] T. Suzuki, Annu. Rev. Phys. Chem. 57, 555 (2006).

[6] C. Z. Bisgaard, H. Satzger, S. Ullrich, and A. Stolow, Chem. Phys. Chem. 10, 101 (2009).

[7] Y.-I. Suzuki, T. Fuji, T. Horio, and T. Suzuki, J. Chem. Phys. 132, 174302 (2010).

[8] N. Pontius, P. S. Bechthold, M. Neeb, and W. Eberhardt, Appl. Phys. B 71, 351 (2000).

[9] J. Stanzel, F. Burmeister, M. Neeb, W. Eberhardt, R. Mitrić, C. Bürgel, and V. Bonačić-Koutecký, J. Chem. Phys. 127, 164312 (2007).

[10] N. Pontius, G. Lüttgens, P. S. Bechthold, M. Neeb, and W. Eberhardt, J. Chem. Phys. 115, 10479 (2001).
[11] V. Blanchet, Z. Zgierski, T. Seideman, and A. Stolow, Nature (London) 401, 52 (1999).

[12] J. R. R. Verlet, A. E. Bragg, A. Kammrath, O. Cheshnovsky, and D. M. Neumark, J. Chem. Phys. 121, 10015 (2004).

[13] A. E. Bragg, J. R. R. Verlet, A. Kammrath, O. Cheshnovsky, and D. M. Neumark, J. Chem. Phys. 122, 054314 (2005).

[14] Y. D. Kim, M. Niemietz, P. Gerhardt, F. von Gynz-Rekowski, and G. Ganteför, Phys. Rev. B 70, 035421 (2004).

[15] H. Häkkinen, M. Moseler, and U. Landman, Phys. Rev. Lett. 89, 033401 (2002).

[16] S. Gilb, K. Jacobsen, D. Schoos, F. Furche, R. Ahlrichs, and M. M. Kappes, J. Chem. Phys. 121, 4619 (2004).

[17] R. Mitrić, J. Petersen, and V. Bonačić-Koutecký, Phys. Rev. A 79, 053416 (2009).

[18] R. Mitrić, J. Petersen, M. Wohlgemuth, U. Werner, V. BonačićKoutecký, L. Wöste, and J. Jortner, J. Phys. Chem. A 115, 3755 (2011). 
[19] P. G. Lisinetskaya and R. Mitrić, Phys. Rev. A 83, 033408 (2011).

[20] J. P. Perdew, K. Burke, and M. Ernzerhof, Phys. Rev. Lett. 77, 3865 (1996).

[21] A. Schäfer, C. Huber, and R. R. Ahlrichs, J. Chem. Phys. 100, 5829 (1994).

[22] D. Andrae, U. Häussermann, M. Dolg, and H. Preuss, Theor. Chem. Acta 77, 123 (1990).
[23] R. Mitrić, U. Werner, and V. Bonačić-Koutecký, J. Chem. Phys. 129, 164118 (2008).

[24] U. Werner, R. Mitrić, and V. Bonačić-Koutecký, J. Chem. Phys. 132, 174301 (2010).

[25] W. Huang, R. Pal, L. M. Wang, X. C. Zeng, and L. S. Wang, J. Chem. Phys. 132, 054305 (2010).

[26] J. Stanzel, Ph.D. thesis, Technische Universität Berlin, 2007. 